4. STELLAR X-RAY SOURCES 


\author{
Richard McCray \\ Joint Institute for Laboratory Astrophysics \\ University of Colorado and National Bureau of Standards \\ Boulder, CO 80309-0440 USA
}

\begin{abstract}
Compact binary $X$-ray sources include white dwarfs, neutron stars, and black holes that are accreting matter from a companion star. The X-ray emission from these systems is produced by the accreting matter as it flows through an accretion disk and strikes the surface of the compact object. The emitting regions have opacities dominated by electron scattering, and radiation pressure is likely to play an important role in the hydrodynamics. Strong magnetic fields greatly modify the hydrodynamics and radiation transfer in the pulsating neutron star sources. Accretion disks have complex structure, including an electron scattering corona, a cool outer region, and possibly a thick torus in their inner region. The structure and stability properties of accretion disks are only partially understood. Major problems exist with the interpretation of the spectra and luminosities of the $X$-ray burst sources. The pulsed $X$-ray emission from the pulsating binary $X$-ray sources probably comes from "mounds" of accreting gas at the magnetic poles of neutron stars, in which the accreting matter is decelerated by radiation pressure. The physics of these systems is reviewed, with an emphasis on problems for which hydrodynamical simulations may be especially useful.
\end{abstract}

\title{
I. INTRUDUCTION
}

The study of compact binary $X$-ray sources became a major enterprise in astrophysics only after the 1969 launch of the UHURU satellite, which quickly yielded the discovery of the pulsating binary $X-$ ray sources Cen $X-3$ and Her $X-1$, which we now know to be accreting neutron stars, and Cyg $X-1$, which we believe to be a black hole. These insights became possible by virtue of the UHURU capability for observing the remarkable variability of the $X$-ray emissions from these sources. Before UHURU, our best clue to the nature of the sources was the brilliant conjecture by SHKLOVSKY [1] that Sco $X-1$ was an accreting neutron star. Although we still lack clear evidence that Sco $X-1$ is a neutron star, most astronomers now recognize that Shklovsky was probably right.

Now we have observed a dazzling array of phenomena in these sources and we are blessed (or cursed!) with many detailed theoretical models for them. I think that we probably have gained some good general ideas about the nature of the $x$-ray emitting regions, but we have not been very successful in quantitatively describing the time variability and spectra of the sources. Perhaps one of the main problems is the lack of tools to deal with time-dependent multi-dimensional flows, which forces theorists to model the emitting regions with stationary flows, perhaps quite inappropriately. At any rate, it is clear that compact $X$-ray sources provide fascinating challenyes in radiation hydrodynamics for those who dare to construct numerical models of the sources.

In this review, I shall begin with a brief overview of the observed properties of the various types of compact X-ray sources. Then I shall discuss some characteristics of the interactions of $X$ rays with matter that are likely to be important 
in these environments. Finally, I shall discuss a number of specific problems in modeling compact $X$-ray sources, stressing those in which (I hope) detailed hydrodynamic studies may provide much needed insights.

Because my topic is so broad, I shall refer to review articles whenever possible and to primary references only for more recent work. For more extensive references, see $[2,3]$.

\section{PHENUMENULOGY OF COMPACT BINARY X-RAY SOURCES}

All of the luminous $10^{34} \lesssim L_{x} \lesssim 10^{39}$ ergs $s^{-1}$ compact galactic $X$-ray sources may consist of a more-or-less normãl star which is transferring matter onto a white dwarf, a neutron star, or a black hole. There may be other types of systems lurking in the few hundred known sources, but we have no clear evidence that this is so. Within each of these three broad cateyories, there are distinctly different types of sources. Schematic $X$-ray spectra of some prototypes are illustrated in Fig. 1.

The accreting white dwarf systems [4-6] with $10^{34} \leqslant L_{x} \lesssim 10^{35}$ ergs s-I can be divided into two types, depending on magnetic field sĩtreñth. The cataclysmic variables, represented by $S S$ Cygni, seem to have low ( $B<10^{5} \mathrm{G}$ ) surface magnetic fields. We believe that mass flows onto the white dwarf through an accretion disk that may actually contact the white dwarf surface in an unstable boundary layer. The nova-like outbursts of these systems may result from a thermal-viscous instability that causes the disk to flush its accumulated gas [7-10]. Cataclysmic variables also display quasi-periodic oscillations during outburst with periods $10 \mathrm{~s}$.

The second class of accreting white dwarf systems, represented by AM Her, have magnetic fields strong enough $\left(>10^{7} \mathrm{G}\right)$ to lock the white dwarf into corotation with its orbit. We believe that these systems have no accretion disk and that the mass flows from the primary star onto a magnetic pole of the white dwarf along magnetic

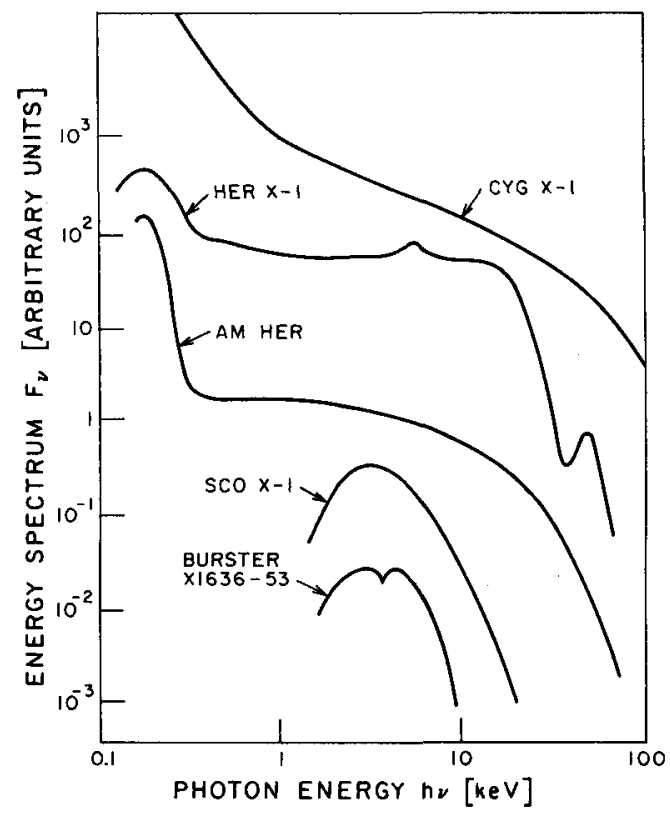

Figure 1: Energy spectra of prototype binary X-ray systems. 
flux lines connecting the two stars. The spectrum of AM Her (Fig. 1) consists of two components with roughly comparable luminosities, one with a color temperature $\mathrm{KT} \sim 40 \mathrm{eV}$ and the other resembling thermal bremsstrahlung from an optically thin gas with temperature $k T \sim 30 \mathrm{keV}$.

The neutron star binary systems [3] can also be divided into two main classes. The Population I systems have massive companion stars. With only one possible exception ( 40 1700-37), the $X$ rays pulsate (with periods ranging from $0.7 \mathrm{~s}$ to $14 \mathrm{~m}$ ) and have relatively hard ( $\mathrm{KT} \sim 10-40 \mathrm{keV}$ ) and complex spectra. The spectrum of Her $x-1$ (the least massive system of this class) is representative. We believe that the $X$-ray emission from such a system comes from the magnetic poles of a rotating neutron star with a strong $\left(1010<B<10^{13} \mathrm{G}\right)$ surface magnetic field. Indeed, the spectruin of Her $x-1$ shows structure at̃ $\sim 50 \mathrm{keV}$ that may be due to electron cyclotron resonance in a magnetic field $B \approx 5 \times 10^{12} \mathrm{G}$.

The $X$-ray sources associated with low mass Population II stars found in the galactic bulge and in globular clusters do not pulse (with the exception of 4U 1626-67). Their spectra, represented in Fiy. 1 by Sco $X-1$, are dominated by a relatively soft ( $k T \sim 2-4 \mathrm{keV}$ ) component resembling a blackbody, and possibly a tail of harder $x$ rays. The $X$-ray emission from a few of these sources, including Sco $X-1$, exhibit remarkable 10-50 Hz quasi-periodic oscillations [11] in which the oscillation frequency is correlated with source luminosity. We believe that the Pop. II X-ray binary systems contain neutron stars with relatively low magnetic fields. Their spectra are soft because the $X$-ray emission is spread out over the neutron star surface and is not confined to the magnetic poles, or because it is reprocessed by optically thick gas near the neutron star.

A substantial fraction, say $30 \%$, of the Pop. II systems display "bursts," in which the luminosity increases suddenly by factors 100 for timescales ranging from a few seconds to minutes $[3,12]$. The spectrum of the bursts resembles that of the non-bursting sources. We believe that the bursts are thermonuclear explosions of hydrogen/helium-rich matter that has accumulated on the neutron star surface. In one source (X1636-53) the TENMA satellite has detected a remarkable absorption feature at $4.1 \mathrm{keV}$; it has been tentatively identified as a gravitationally redshifted $6.7 \mathrm{keV} \mathrm{Fe} \mathrm{K}$ absorption line from the neutron star surface [13].

Finally, we know a few binary $x$-ray systems, including Cyg $x-1$, IMC $x-3$, LMC $x-1$, and $A$ 0620-00, which we believe to contain black holes. Cyg $x-1$ is the best known. Its $X$-ray emission fluctuates randomly with large amplitude on timescales ranging from $10^{-3} \mathrm{~s}$ to months, and its spectrum (Fig. 1) is distinguished from the spectra of the neutron star and white dwarf sources by its concave shape at low energy and its hard ( 100 keV) tail.

\section{BASIC PHYSICS OF ACCRETION FLLWS}

(a) The Eddington Limit

Radiation pressure plays a fundamental role in limiting the luminosity of accreting $x$-ray sources. The net attractive force per unit mass due to a compact object of mass $M_{\star}$ on an optically thin gas stream $i 1 l$ uminated by an $X$-ray flux $L_{x} / 4 \pi r^{2}$ is given by $F=\operatorname{Gim}_{\star}\left(1-L_{x} / L_{\star}\right) / r^{2}$, where

$$
L_{\star}=\Omega_{r} G_{\star} m_{H} / k=L_{E}\left[\frac{\Omega^{2}}{4 \pi}\right]\left[\frac{\kappa}{\kappa_{e}}\right]
$$

and

$$
L_{E}=2.5 \times 10^{38} \mathrm{ergs} \mathrm{s}^{-1}\left[\frac{M_{\star}}{M_{\theta}}\right]\left[1+\chi_{H}\right]^{-1} \text {. }
$$

The factor $\Omega_{R} / 4 \pi$ accounts for beaming of the emitted radiation and the factor $k / \kappa_{e}$ 
allows for the possibility that the opacity, $k$, may be different from the Thomson scattering opacity, $k_{e}$. The factor $\left[1+x_{H}\right]-1$, where $X_{H}$ is the fractional abundance of hydrogen atoms, results from the fact that there are approximately two electrons per nucleon in all elements except hydrogen.

\section{(b) Energy Generation}

The time-averaged bolometric luminosity may be related to the time-averaged accretion rate $A$ by

$$
L_{x}=\varepsilon c^{2} M=\left[\frac{\varepsilon}{0.2}\right]\left[\frac{\dot{M}}{10^{-8} M_{0} y r^{-1}}\right] L_{E},
$$

where the factor $\varepsilon \sim 0.05$ to 0.4 for neutron stars and $\varepsilon \sim 10^{-4}$ to $10^{-3}$ for white dwarf stars. There is also a contribution to $\varepsilon$ from thermonuclear burning of accreted matter which ranges from $\sim 10^{-3}$ to $10^{-2}$, depending on the mix of hydrogen and helium in the accreting matter. Note that although the thermonuclear energy release makes a relatively small contribution to the time-averaged $X$-ray emission from neutron stars, it may clearly dominate during $X$-ray bursts.

\section{(c) Upacity}

The emitting regions of the bright compact $X$-ray sources are likely to be optically thick to electron scattering. Assuming that the accretion flow is confined to a cone of solid angle $\Omega_{m}$ and is flowing inward with a velocity $v_{r}$ less than the freefall velocity, $v_{f f}$, we estimate the scattering optical depth to be

$$
\tau_{e} \approx 2 e^{-1 / 2}\left[\frac{4 \pi}{\Omega_{\mathrm{In}}}\right]\left[\frac{v_{\mathrm{ff}}}{v_{r}}\right]\left[\frac{L^{\mathrm{x}}}{L_{E}}\right] \text {. }
$$

\section{(d) Characteristic Temperatures}

There are two obvious characteristic temperatures that may be relevant to accretion flows. The first is the black body temperature, estimated by applying the StefanBoltzmann law at the radiating surface, which may be written

$$
k T_{B B}=k T_{1}\left[\frac{4 \pi}{\Omega_{m}}\right]^{1 / 4}\left[\frac{L^{\prime}}{L_{E}}\right]^{1 / 4},
$$

where $\mathrm{kT}_{1} \approx 1.8 \mathrm{keV}$ for a neutron star or stellar-mass black hole and $\mathrm{kT} T_{1} \approx 60 \mathrm{eV}$ for a white dwarf star. The second characteristic temperature is the one that would result if freely falling gas were shocked adiabatically at the surface of the compact object. It is $k T_{f f} \approx 40 \mathrm{MeV}$ for a neutron star and $k T_{f f} \approx 10-100 \mathrm{keV}$ for a white dwarf star. One would expect the characteristic spectral temperature to be bounded by $T_{B B}$ and $T_{f f}$ unless the observed $X$ rays are absorbed and re-radiated by surrounding matter, but this does not constrain the spectrum very tightly. Remarkably, there is evidence for both temperatures in the spectrum of AM Her (Fig. 1). Its spectrum can be interpreted roughly as the result of emission from a shocked region where the accretion flow strikes the white dwarf surface. The hard $x$ rays come from optically thin emission of the shocked gas, and the soft $X$ rays come from that emission that is directed downward and re-radiated by the white dwarf atmosphere.

\section{INTERACTIUN OF $X$ RAYS AND MATTER}

Here I list a few properties of the interaction of $X$ rays with matter that are likely to be important in the physics of compact $X$-ray sources. 
(a) X-ray Heating of Optically Thin Gas - Thermal Instability

Detailed studies $[14,15]$ show that an optically thin gas of typical cosmic composition that is heated by $X$ rays and cooled by emission of radiation will reach a stationary temperature that is illustrated schematically in Figure 2, where $k T_{X}$ is roughly the average $X$-ray photon energy and the abscissa is given by the ratio of the radiation pressure to gas pressure:

$$
\Xi=\left[\frac{L_{x}}{4 \pi r^{2} c p_{g}}\right] .
$$

The most important qualitative feature of this curve is the thermal instability that occurs for $\Xi \approx 1-10$. This instability has significant consequences for the gas flows in the vicinity of $X$-ray sources. First, $X$-ray heating can suppress or modify accretion flows even when radiation pressure is unimportant, for optically thin gas at distance from the source $r>10^{11} \mathrm{~cm}\left[1 \mathrm{keV} / \mathrm{kT} T_{x}\right]\left[\mathrm{M}_{\star} / \mathrm{M}_{\Theta}\right]$ if $\mathrm{L} \gtrsim 10^{-2} \mathrm{LE}$ [16]. Second, an X-ray source can heat and drive a thermal wind from añy cool atmosphere that it illuminates, for example, the atmosphere of a companion star or the surface of an accretion disk [17].

\section{(b) Radiation Pressure}

Radiation pressure is likely to dominate the hydrodynamics of the emitting regions of compact $X$-ray sources if they are optically thick. For example, if the radiation field is in LTE, the ratio of radiation pressure to gas pressure in the flow is given approximately by

$$
R=P_{\text {rad }} / P_{\text {gas }} * 0.025 \rho^{-1}\left[k T_{\text {eff }} / 1 \mathrm{keV}\right]^{3} \text {, }
$$

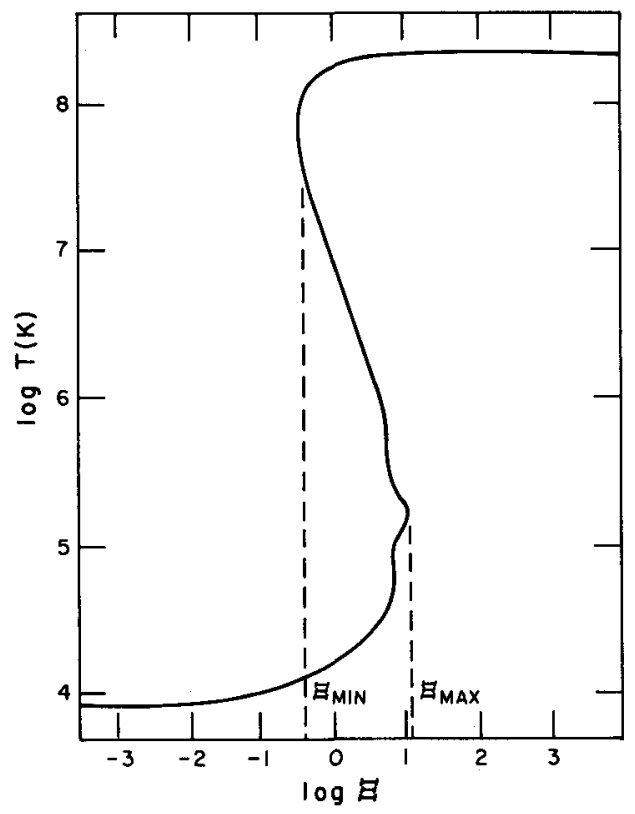

Figure 2: Stationary temperature, $T$, of an optically thin gas exposed to $X$ rays as a function of the ionization parameter, $\mathrm{E}$. The gas must be cool $\left(\sim 10^{4} \mathrm{~K}\right)$ for $\Xi<\Xi_{\min }$ and hot $\left(\sim 10^{8} \mathrm{~K}\right)$ for $\Xi>\Xi_{\max } \cdot$ (Courtesy of Dr. T. Kallman.) 
where $\rho$ is the gas density in $\mathrm{g} \mathrm{cm}^{-3}$. Typically, $R \ll 1$ for the photosphere of compact X-ray sources, even at the surface of a neutron star.

\section{(c) Electron Scattering}

The ratio of (Rosseland mean) electron scattering opacity to free-free opacity is given approximately by

$$
\kappa_{e} / \kappa_{f f} \approx 30 \rho^{-1}\left[k T_{e f f} / 1 \mathrm{kev}\right]^{3.5} .
$$

For typical conditions at $X$-ray photospheres, this ratio is $\gg 1$. As a result, scattering opacity suppresses the emission from $X$-ray photospheres by a factor $\sim\left[\mathrm{ke}_{\mathrm{e}} / \mathrm{kff}\right]^{-1 / 2}$. The fact that this factor is small for typical $X$-ray photospheres has two important implications. First, it implies that the radiation field is photon-starved, so that the color temperature will typically be substantially greater than the effective temperature and the radiation pressure will be less than that indicated by equation (7). Second, it implies that LTE is likely to be a bad assumption for the hydrodynamics of $X$-ray emitting regions; photon kinetics (radiation transfer) will be required.

Another consequence of the dominance of electron scattering opacity is that the main eneryy transfer between the gas and the photons may result from electron recoil during Compton scatteriny -- "Comptonization." The importance of Comptonization in establishing the $X$-ray spectrum can be measured by the parameter

$$
y=4\left[k T_{g} / m_{e} c^{2}\right] \tau_{e}^{2},
$$

where $\tau_{e}$ is the electron scattering optical depth. For example, Comptonization will up-scatter soft photons that propayate into a slab of hot gas. As a result, the spectrum that is reflected from a semi-infinite slab will resemble that from optically thin bremsstrahlung emission, and the spectrum that is transmitted through a slab will resemble a power law for $y<1$ and a Wien spectrum for $y>1$. Comptonization will substantially amplify the luminosity of the transmitted $X$ rays if $y>1$. Because typical $X$-ray emitting regions are photon-starved and Comptonization may be the dominant energy loss mechanism, soft photon sources can play a major role in the hydrodynamics of hot gas in X-ray sources, enhancing the energy emissivity and cooling the gas.

\section{(d) Magnetic Suppression of Opacities}

The strony $(B \geqslant 1011$ G) magnetic fields of the Pop. I neutron star sources can have profound effects on the electron scattering opacities for $X$-ray photon energies [18]. Below and at the cyclotron resonance frequency,

$$
H_{\omega_{\mathrm{g}}}=11.6 \mathrm{keV}\left[\mathrm{B} / 10^{12} \mathrm{G}\right] \text {, }
$$

the magnetic field introduces strony frequency and angular dependence of the electron scattering and bremstrahlung cross sections, distinguishing among the ordinary and extraordinary modes, respectively polarized with electric vectors within and perpendicular to the plane defined by the $B$ and $\mathbf{k}$ vectors. Below resonance the scattering and absorption cross sections for the ordinary mode are reduced (compared to the corresponding cross sections for $B=0$ ) by the factor $\sin ^{2} \theta$, where $\theta$ is the anyle between $B$ and $k$, and the cross sections for the extraordinary mode are reduced by the factor $\left(\omega / \omega_{B}\right)^{2}$. Both modes have resonances in their scattering and absorption cross sections, increasing by a few orders of magnitude within a few Doppler widths of $\omega_{B}$. Above $\omega_{B}$ the cross sections approach their $B=0$ values.

This modification of the opacities by strong magnetic fields must be partly resuonsible for the beaming of the $X$-ray emission from the pulsating Pop. I sources, but the details of how this works are still not clear, because we still do not have a good understanding of the hydrodynamics of the emitting regions (ćf. $\S V I I$ ). 


\section{ACCRETIUN DISKS}

Now I shall discuss some basic structures that are believed to be important in accretion flows, emphasizing outstanding observational puzzles and unsolved or partially solved theoretical problems where I believe that numerical experiments in radiation hydrodynamics may contribute substantial insight.

In most binary $X$-ray sources the accreting gas flows into a compact object through a centrifugally supported accretion disk [19]. (The exceptions are the AM Her stars and, possibly, some Pop. I neutron star binaries in which the strong stellar wind of the companion star might have too little angular momentum to form a disk.) Despite the elegant theories for their structures, I think that accretion disks are not very well understood. It is clear, however, that they are complex structures with different physical effects prevailing in different regions.

\section{(a) Accretion Disk Coronae}

A few years ago, observers noticed spectrally independent partial $X$-ray eclipses in the Pop. II binary sources $4 \mathrm{U} \quad 1822-37$ and $4 \mathrm{U} 2129+47$ [20]. These observations imply that these sources must be extended, with dimensions $>10^{10} \mathrm{~cm}$, even though the $X$ rays must be produced near the surface of a neutron star. We infer that the $X$ rays have been scattered by electrons in a highly ionized corona above the accretion disk [21-23]. These accretion disk coronae are believed to be manifestations of the therial instability described above in $\S$ IIIa. The corona is maintained at a temperature $\sim 10 \mathrm{keV}$ by the $X$-rays from the compact source. The $X$ rays are scattered by the corona, which has a typical optical depth $\tau_{e} \sim 0.3$, and this scattering causes the relatively cool disk photosphere to be illuminated indirectly by the primary $X-$ rays. The scale height, $z$, of the corona increases with increasing radial distance, $r$, from the source, roughly as $z \propto r^{3 / 2}$, but it collapses at a radial distance where the scattering optical depth along the line-of-sight to the central source becomes substantial and the hot phase cannot be maintained by the $X$-ray heating. The optical depth of the corona depends on the primary $X$-ray luminosity, $L_{X} ; L_{x} \approx 0.03 L_{E}$ is required to explain the observations. With such a dimension and $k T^{x} * 10 \mathrm{keV}$, the outer part of the accretion disk corona is likely to be the source of substantial mass loss in a hot wind, perhaps even enough to burn away the disk before it can be replenished.

The accretion disk corona is an excellent candidate for a numerical simulation. The hydrodynamics is dominated by gas pressure, but the temperature is controlled by the radiation field, which depends on the global flow. The trick is to find an efficient way to combine the two-dimensional hydrodynamical calculation with a calculation of the radiation transfer. The problem is well-posed, the basic physics is straightforward, and clever analytic studies have provided a good basic understanding of the structure of the hydrostatic part of the corona. However, it is difficult to model analytically the transonic part of the corona, and numerical models might reveal some interesting surprises, such as poloidal circulation flows.

\section{(b) The Inner Disk}

The accretion disk is probably disrupted within a "magnetopause" radius, $R_{M}$, that may be estimated roughly by equating the dynamic pressure of the accretion flow to the magnetic stresses:

$$
K_{M} \approx 2 \times 10^{8} \mathrm{~cm}\left[L_{L} L_{E}\right]^{-2 / 7}\left[B_{0} / 10^{12} \mathrm{G}\right]^{4 / 7},
$$

where $\mathrm{B}_{0}$ is the dipole field strength at the surface of the neutron star [24]. Thus, the Pop. I sources, with $B_{0} \gtrsim 10^{11} \mathrm{G}$, may have only a relatively cool outer disk and no inner disk. However, black noles will have a "disk" that extends into the innermost stable orbit, and a Pop. I neutron star source with B< $10^{8} \mathrm{G}$ will have an inner "disk" that extends to the neutron star surface. 
I write "disk" in quotes here because we know so little about the structure of the inner regions of accretion disks. We do know that the inner, optically thin, reyions of classical model accretion disks are subject to a variety of thermal and viscous instabilities when the radiation pressure becomes comparable to the gas pressure. Furthermore, this inner region may have a thickness comparable to its radius, so that it is better called a torus than a disk. A disk can thicken into a torus for two reasons. First, in relatively low luminosity sources, the density of the inner disk may be low enough that the ions (which do not radiate) do not effectively transfer their thermal energy to the electrons (which do). Then an "ion torus" develops, in which the ion thermal velocity is comparable to the Kepler velocity, while the electron temperature may be much less than the ion temperature $[25,26]$. Second, a disk can become thick because its internal radiation pressure becomes comparable to or greater its gas pressure $[27,28]$. This situation will obtain when the disk luminosity becomes comparable to $L_{E}$. Moreover, a torus is subject to non-local instabilities that grow on dynamical timescales $[29,30]$, so the significance of stationary models for tori (in which this instability is not permitted) is not clear.

There are plenty of observations whose interpretation begs for a better understanding of the nature of these inner regions of accretion disk/tori. First, it seems likely that the wild intensity fluctuations of black hole sources such as Cyg $X-1$ are a manifestation of non-linear instabilities in the inner disk flow. Second, the quasi-periodic outbursts of the cataclysmic variables and of the "rapid burster" may be due to the non-linear development of a thermal-viscous instability in the disk $[7-10,31]$. Third, the newly-discovered quasi-periodic oscillations in some of the Pop. II neutron star sources may require an instability to make the inner disk break up into "blobs" that orbit with the Kepler period [32].

Another general consideration to bear in mind when interpreting observations of compact $X$-ray sources is that these tori, if they exist, will likely collimate most of the $X$-ray luminosity into a relatively small solid angle, so that we cannot confidently infer the $X$-ray luminosity of a source from its observed intensity and a known distance by assuming an isotropic source. Thus, it is possible that many of the sources whose luminosities appear to be less than $L_{E}$ actually have primary accretion luminosities close to $L_{E}$.

\section{X-RAY BURST SOURCES}

It would seem that the $X$-ray burst sources present one of the most straightforward problems in radiation hydrodynamics. According to the standard model [3], the bursts are a manifestation of thermonuclear flashes of hydrogen and helium gas that has accreted onto the surface of a weakly magnetized neutron star. Thus, it is reasonable to attempt to describe the bursts with a spherically symmetric hydrodynamic model. Since $X$-ray burst sources have been discussed in these proceedings by $D r$. MELIA [12], I shall only remark on a few outstanding problems related to the interpretation of their spectra.

First, it is clear that the $X$-ray bursters do not radiate as blackbodies. One can estimate that even if the neutron star atmosphere is in hydrostatic equilibrium, a typical observed $X$-ray photon suffers several electron scatterings before it escapes the atmosphere. Thus, although the emergent spectrum may resemble a blackbody, it is actually a Comptonized dilute blackbody spectrum with color temperature $T_{c}>[1.5-2] T_{\text {eff }}[33,34]$. The observations support this conclusion. Indeed, if it is assumed that a neutron star surface radiates as a blackbody at its Eddington limit, general relativistic effects (light bending and gravitational redshift) rule out a color temperature $k T_{C}>2 \mathrm{keV}$ for any known neutron star model. Yet some bursters have $\mathrm{kT}_{\mathrm{C}}>3 \mathrm{keV}$.

Second, the spectra of some events, the so-called "flat-top" bursts, strongly suggest expansion of the neutron star photosphere -- perhaps in a neutron star wind. In these events, the burst luminosity appears to rise rapidly to $\sim L_{E}$, then level of $f$ 
for some $10-100 \mathrm{~s}$ before declining again. During the rise phase, $\mathrm{T}_{c}$ drops below $2 \mathrm{keV}$ while $L_{X} \sim L_{E}$. If the photosphere radiated as a blackbody, a photospheric radius $R_{p}>30 \mathrm{~km}$ would be required to explain such a low $T_{C}$. Allowing for the fact that $T_{c} S T_{\text {eff }}$ requires an even larger $R_{p}$ to explain the low $T_{c}$.

Third, it seems to be impossible to construct a model for a thermonuclear flash on a neutron star that will radiate $X$ rays with a luminosity substantially greater than $L_{E}$. The problem is that such a luminosity must be generated by thermonuclear burning far below the photosphere, and if the nuclear luminosity exceeds $L_{E}$ it will lift the atmosphere on a dynamical time of less than a millisecond, converting the excess luminosity to kinetic eneryy of the expanding atmosphere. Detailed models [cf. 12] show that the maximum possible luminosity that can be sustained is only a few percent greater than $L_{E}$.

Fourth, this theoretical result lends strong support to the use of X-ray bursters as standard candles. Then, a remarkable result emerges. If it is assumed that the $X$-ray bursters that are concentrated in the direction of the galactic center have luminosities < $L_{\epsilon}$, the distance to the galactic center must be less than $6 \mathrm{kpc}$ [35]. This conclusion is hard to avoid, even though it has dramatic implications for galactic structure.

Fifth, the interpretation of the $4.1 \mathrm{keV}$ absorption feature in the spectrum of the $X$-ray burster $X B \quad 1636-53$ as a gravitationally redshifted iron line [13] is very problematical. The maximum possible gravitational redshift from the surface of any published stable neutron star model is about $\mathrm{z}_{\mathrm{g}} \approx 0.60$, and this is the value of $z_{g}$ that is required to redshift the $6.7 \mathrm{keV} \mathrm{Fe}+24 \quad R_{\alpha}$ line to the observed $4.1 \mathrm{keV}$. However, a simple estimate shows that, during a burst, any $\mathrm{Fe}$ in the atmosphere of the neutron star would be almost fully ionized, with perhaps a little $\mathrm{Fe}^{+25}$ but negligible $\mathrm{Fe}^{+24}$. There is no stable neutron star model with sufficient gravitational redshift $\left(z_{\mathrm{g}}=0.7\right)$ to shift the $6.97 \mathrm{keV} \mathrm{Fe}{ }^{+25} \mathrm{~K}_{\alpha}$ line to $4.1 \mathrm{keV}$.

\section{vil. NEUTKON STAR POLAR CAPS}

The pulsed emission from the Pop. I binary sources must come from hot spots on rotating neutron stars, presumably magnetic poles to which the accretion flows are funneled by the strong maynetic fields. For some sources, e.g., Her $X-1$, the $X$-ray pulse profiles are rather simple, characterized by a broad non-symmetric double pulse with a profile that depends weakly on spectral energy. For others, e.g., 40 0900-40, the pulse profiles are quite complex and energy-dependent [36]. Perhaps in sources of the latter type the dipole component of the neutron star does not dominate, so that the $X$-ray emission comes from accretion onto several magnetic poles of different strenyths. But even in the simpler sources, it is a major challenge to produce a plausible model that accounts for the pulse profile and its spectrum. Despite intensive theoretical work spanning more than a decade, we are still not sure, for example, whether the emission from one of these polar caps is produced in a fan beam or a pencil beam, or how the beam is collimated. The problem remains a major challenge of theoretical astrophysics.

The pioneering papers by DAVIDSON [37] and BASKO and SUNYAEV [38-40] are still probably the best introduction to the physics involved in this problem.

We can gain some insight by making dimensional estimates, taking Her $x-1$ as a prototype. It has a time-averaged luminosity $L_{x} \approx 2 \times 10^{37}$ ergs $\mathrm{s}^{-1}$ and a surface magnetic field $\mathrm{B}_{0} \sim 5 \times 10^{12} \mathrm{G}$ (interpreting the $50 \mathrm{keV}$ spectral feature as a gravitationally redshifted cyclotron line). Assuming that the accretion flow does not attach to dipole field lines that close inside of $R_{M}[e q .(11)]$, we may estimate that when it reaches the neutron star surface it is confined to a polar angle

$$
\theta_{0}=0.1\left(\frac{L_{X}}{L_{E}}\right)^{1 / 7}\left[\frac{B_{0}}{10^{12} G}\right]^{-2 / 7} \text {. }
$$


Then, assuming that the flow uniformly fills the accretion column, we may estimate the density of freely falling gas above the polar cap and the electron scattering optical depth (ordinary mode) parallel and perpendicular to the accretion column. They are, respectively, $\rho=10^{-3} \mathrm{~g} \mathrm{~cm}^{-3}, \tau_{\|} \approx 10^{3}$, and $\tau_{f} \approx 20$ [the opacities in the extraordinary mode are less by factors $\left.\sim\left(h_{\omega} / 50 \mathrm{keV}\right)^{2}\right]$. The effective Eddington limit for the polar cap [eq. (1)] is approximately $L_{\star} \approx L_{E}\left(\theta_{0}^{2} / 4\right)\left(\mathrm{ke}_{\mathrm{e}} / \mathrm{k}\right)=5 \times 10^{35}$ ergs $\mathrm{s}^{-1}$. Since the observed luminosity of the beam appears to be many times this value, we infer that radiation pressure strongly affects the flow in this region. We may also estimate the blackbody temperature [eq. (5)] of the polar caps of Her $X-1$ to be $\mathrm{KT}_{\mathrm{BB}} \sim 8 \mathrm{keV}$, substantially less than the observed color temperature (Fig. 1) of $k T_{C} \sim 20 \mathrm{keV}$.

In order to construct models for the beamed X-ray emission, we must solve the coupled problem of the radiation hydrodynamics of the flow and the transfer of radiation in the strongly anisotropic and birefringent medium. Not surprisingly, this has not yet been done. Most theoretical calculations to date have addressed model problems in which only half of the actual problem is solved while the other half is replaced by an artificial assumption. For example, if the radiative transfer is solved in detail, the hydrodynamics problem is replaced by the assumption of an isothermal plane-parallel slab or a uniform density cylinder; or, if the hydrodynamics is solved, the radiative transfer problem is approximated by LTE and isotropic diffusion.

If the accretion rate is low, such that $L_{<} L_{\star}$, it would be reasonable to neglect the effect of the radiation on the hydrodynamics. In that case, the accretion flow i.s stopped and thermalized by collisions of the infalling protons in a thin $(\sim 1 \mathrm{~m})$ slab of fairly dense $\left(\rho \sim 1 \mathrm{~g} \mathrm{~cm}^{-3}\right)$ gas at the neutron star surface, with Thomson scattering optical depth $\tau_{e} \sim 20$. Adopting such a "slab model" greatly simplifies the theoretical problem; but, unfortunately, it is not valid to neglect radiation pressure for most of the known pulsating $X$-ray binaries.

Detailed calculations have been made for the transfer of $X$ rays through isothermal slabs and filled cylinders in very strong magnetic fields, including all the nasty details of polarization, angular dependence, resonances, and non-coherent scattering $[18,41]$. The main qualitative theoretical results are: (1) the cyclotron resonance generally appears as an absorption feature; and (2) the emitted radiation is beamed perpendicular to the surface, i.e., a slab produces a pencil beam and a cylinder produces a fan beam. Some of the calculated pulse profiles agree remarkably well with the observed ones, but I do not take this agreement too seriously, given the number of adjustable parameters and the artificiality of the assumptions in the theory. Even so, these calculations provide vital insight into the physics of the spectral formation, and it would be very interesting to see how the theoretical results would change if strong temperature gradients were assumed in the $X$-ray photospheres.

The known Pop. I binary $X$-ray pulsars have observed luminosities $L>L_{\star}$, so that the accretion flow onto the magnetic pole of the neutron star is probably decelerated by radiation pressure. Thus, a realistic theoretical model for such a source must include a calculation of the hydrodynamic structure of such an accretion "mound." This problem has been addressed most recently by ARONS, KLEIN and LEA [42] following earlier work by DAVIDSON [37], WANG and FRANK [43], and KIRK [44].

The model embodies the following assumptions: (1) the accreting gas flows along riyid magnetic field lines (a safe assumption); and (2) the accretion column is uniformly filled (for simplicity). In the hydrodynamic calculation, the radiative transfer is solved in diffusion approximation, allowing for different diffusion coefficients along and perpendicular to the magnetic field.

With these assumptions, the model can be described by four coupled equations: the equations of continuity, Euler's equation, an energy equation, and a diffusion equation. Terms accounting for the enthalpy flux of radiation advected with the gas flow appear in the Euler equation and the diffusion equation. The strategy is first 
to solve these equations with a two-dimensional hydrodynamics code, then to use the solution for the gas structure as a basis for a more detailed radiative transfer calculation which does not rely on the diffusion approximation. One hopes that this strategy will provide a more realistic model for the $X$-ray beam.

One can construct an analytical model that provides valuable insight into the structure of the accretion mound $[42,44]$. To do this. one makes the further approximations that the perpendicular and parallel diffusion coefficients are constant (but not necessarily equal), that the gas is decelerated suddenly by radiation at a sharp front, and that the gas is supported by radiation pressure in approximate hydrostatic equilibrium in the mound beneath this front. Then the equations become separable; in the resulting solution the height of the mound boundary has a parabolic shape:

$$
h \approx R_{\star}\left[\frac{L_{x}}{L_{E}}\right]\left[1-\left(\frac{\theta}{\theta_{c}}\right)^{2}\right] \text {, }
$$

where $R_{\star}$ is the neutron star radius, $\theta_{c}$ is the co-latitude of the polar cap, $L_{c}$ is the actual accretion luminosity of the polar cap and $L_{F}$ is the classical Eddington luminosity [ey. (2), not eq. (1)]. Numerical calculations [42] verify the qualitative features of this model and indicate, as expected, that the radiation emerges from the side of the mound in a fan beam.

While I believe that these calculations are the most promising approach so far to understanding the emitting regions of the pulsating $X$-ray sources, I feel obliged to mention major obstacles that confound the quest for even a qualitative understanding of the actual beaming mechanism. First, the observations tell us that the $X$-rays are not emitted in an axisymmetric fan beam; otherwise the pulse profiles would have a mirror symmetry. Second. the approximation that the freely falling gas is distributed uniformly over the polar magnetic field lines may be grossly incorrect. This distribution may well control whether the beam is a pencil or a fan; for example, if most of the infalling gas is confined to a hollow cone rather than a filled cylinder much of the radiation may emerge from the interior of the cone in a pencil beam [40]. Unfortunately, it may be very difficult to guess this distribution, since it is established by MHD instabilities that occur in the magnetosphere, far from the polar cap; understanding this region is another major theoretical problem $[24,45]$.

I conclude this section with a speculation addressed to the following question: why do the pulsating $X$-ray sources tend to have flat spectra with rather sharp cutoffs? We might find a clue to this in the putative cyclotron resonance structure at $\sim 50 \mathrm{keV}$ in the spectrum of Her $X-1$. My idea. unsupported by any detailed calculation, is this: perhaps the $50 \mathrm{keV}$ feature is not the cyclotron resonance frequency, but the first harmonic. Then the fundamental is at $\sim 25 \mathrm{keV}$, roughly at the knee of the $X$-ray spectrum where most of the $X$-ray power is emitted. The advantage of this model is that the $25 \mathrm{keV}$ spectral temperature of Her $X-1$ can be explained naturally as the result of a thermostatic control by copious cyclotron radiation that sets in when $k T \sim n_{\omega_{B}}$, much as atomic line cooling provides a thermostat setting the temperature of diffuse interstellar gas at $\sim 10^{4} \mathrm{~K}$. The problem with the model is to explain why the spectrum should fill in and become flat below the resonance at $25 \mathrm{keV}$.

\section{SUMARY AMD CONCLUSIONS}

I have been very much impressed at this conference to learn of the enormous potential of supercomputers to simulate problems in radiation hydrodynamics. In this review I have tried to show that compact $X$-ray sources provide ideal turf for supercomputer jocks to display their prowess.

I was also much impressed by a conversation I had several months ago with KarlHeinz Winkler, in which he said that he planned to make his adaptive mesh code 
work like a "video game," whereby one could put his favorite physics and boundary conditions into any one-dimensional problem and watch the solution develop in lurid false-color. This is a very exciting prospect to me (with my PC mentality), and I very much look forward to the time when I can go into the neighborhood supercomputer arcade to play video games with binary $X$-ray sources. Here are the ones that I would like to play first:

1. "Shining Crown" -- warm up with an adventure game, and see the beautiful corona of an accretion disk gently merge into a wind. Probably a fairly easy and peaceful game, but watch out for surprises.

2. "Splash" -- if you have a taste for more violent action, play with a torrent of gas falling at $20 \%$ of the speed of light onto the magnetic pole of a neutron star. See how the $X$-ray beam changes as you change the distribution of the infalling gas on the magnetic field lines.

3. "Torus" -- for experts only. Be prepared to respond on millisecond timescales to the furious instabilities of the $i$ on torus and the radiation torus. Be careful: if you start playing this one, you may never escape!

This work was supported by the NASA Astrophysical Theory Program under grant NAGW-766. I am grateful to Dimitri Mihalas for inviting me to this meeting, which I enjoyed very much, and for his encouragement and forbearance.

\section{REFERENCES}

1. 1. S. Shklovsky: Sov. Astr.-AJ 11, 749 (1968).

2. S. S. Holt and R. McCray: Ann. Rev. Astr. Ap. 20, 323 (1982).

3. P. C. Joss and S. A. Rappaport: Ann. Rev. Astr. Ap. 22, 537 (1984).

4. J. Liebert and H. S. Stockman: in Cataclysmic Variables and Low Mass X-Ray Binaries, edited by $\mathrm{D} . \mathrm{Q}$. Lamb and J. Patterson (Reidel, Dordrecht, 1985), pp. 151-178.

5. D. Q. Lamb: in Cataclysmic Variables and Low Mass X-Ray Binaries, edited by D. Q. Lamb and J. Patterson (Reidel, Dordrecht, 1985), pp. 179-218.

6. B. Warner: in Cataclysmic Variables and Low Mass X-Ray Binaries, edited by

D. Q. Lamb and J. Patterson (Reidel, Dordrecht, 1985), pp. 269-280.

7. J. Faulkner, U. N. C. Lin, and J. C. B. Papaloizou: M.N.R.A.S. 205, 359 (1983).

8. F. Meyer and E. Meyer-Hofmeister: Astr. Ap. 128, 420 (1983).

9. J. C. B. Papaloizou, J. Faulkner, and D. N. C. Lin: M.N.R.A.S. 205, 487 (1983).

10. D. N. C. Lin, J. C. B. Papaloizou, and J. Faulkner: M.N.R.A.S. $\overline{212}, 105$ (1985).

11. W. G. H. Lewin and J. van Paradijs: Comments Ap. (1986), in press.

12. F. Melia and P. C. Joss: these proceedings (1986).

13. I. Waki, I. et al.: Publ. Astr. Soc. Japan 36, 819 (1984).

14. J. H. Krolik, C. F. Mckee, and C. B. Tarter: Ap. J. 249, 422 (1981).

15. T. Kallman and R. McCray: Ap. J. Suppl. 50, 263 (1982).

16. J. P. Ostriker, R. McCray, R. Weaver, and A. Yahil: Ap. J. Lett. 208, L61 (1976).

17. R. London, R. McCray, and L. H. Auer: Ap. J. 243, 970 (1981).

18. P. Meszaros and W. Nagel: Ap. J. 298, 147 (1985).

19. J. E. Prinyle: Ann. Rev. Astr. Ap. 19, 137 (1981).

20. N. E. White and S. S. Holt: Ap. J. 257. 318 (1982).

21. M. C. Begelman and C. F. McKee: Ap. J. 271, 89 (1983).

22. M. C. Begelman, C. F. McKee, and G. A. Shields: Ap. J. 271, 70 (1983).

23. G. A. Shields, C. F. Mckee, D. N. C. Lin, and M. C. Begelman: Ap. J. (1985), in press.

24. F. K. Lamb: in AIP fonference Proceedings No. 115, High Energy Transients in Astrophysics, edited by S. E. Woosley, (American Institute of Physics, New York, 1984), Pp. 175-215.

25. S. L. Shapiro, A. P. Lightman, and D. M. Eardley: Ap. J. 204, 187 (1976).

26. M. Rees, M. C. Begelman, R. D. Blandford, and E. S. Phinney: Nature 295, 17 (1982). 
27. B. Paczynski and P. Wi ita: Astr. Ap. 88, 23 (1980).

28. K. Hoshi: Prog. Th. Phys. Suppl. 70, 181 (1981).

29. J. C. B. Papaloizou and J. E. Pringle: M.N.R.A.S. 208, 721 (1984).

3U. J. C. B. Papaloizou and J. E. Pringle: M.N.R.A.S. $\overline{213}, 799$ (1985).

31. R. E. Taam and D. N. C. Lin: Ap. J. 287. 761 (1984).

32. F. K. Lamb, N. Shibazaki, J. Shaham, and M. A. Alpar: Nature 317. 681 (1985).

33. K. A. London, R. E. Taam, and W. M. Howard: Ap. J. Lett. 287, L27 (1984).

34. R. A. London, W. M. Howard, and R. E. Taam: Ap. J. (1986), in press .

35. T. Ebisuzaki, T. Hanawa, and D. Sugimoto: Publ. Astron. Soc. Japan 35, 17 (1983).

36. N. E. White, J. H. Swank, and S. S. Holt: Ap. J. 270, 711 (1983).

37. K. Davidson: Nature Phys. Sci. 246, 1 (1973).

38. M. M. Basko and R. A. Sunyaev: Sov. Phys. JETP 41, 52 (1974).

39. M. M. Basko and R. A. Sunyaev: Astr. Ap. 42, 311 (1975).

40. M. M. Basko and R. A. Sunyaev: M.N.R.A.S. 175, 395 (1976).

41. P. Meszaros and W. Nage1: Ap. J. 299, $138 \overline{(1975)}$.

42. J. Arons, R. I. Klein, and S. M. Lea: Ap. J. (1986), submitted.

43. Y. M. Wany and J. Frank: Astr. Ap. 93, 255 (1981).

44. J. G. Kirk: Astr. Ap. 142, 430 (1985).

45. J. Arons. D. J. Burnard. R. I. Klein. C. F. McKee. R. Pudritz. and S. M. Lea: in AIP Conference Proceedings No. 115, High Energy Transients in Astrophysics, edited by S. E. Woosley, (American Institute of Physics, New York, 1984), pp. 215-234. 